\title{
On the spin distribution in bridged anthracene-viologen molecules: an electron-nuclear double resonance study
}

\author{
By N. S. SARICIFTCI $\dagger$, A. WERNER, A. GRUPP and M. MEHRING \\ 2 Physik Institut, Universtat Stuttgart, Pfaffenwaldring 57, 7000 Stuttgart 80, \\ Germany \\ G. GÖTZ, P. BÄUERLE and F. EFFENBERGER \\ Institut für Organische Chemie, Universtat Stuttgart, Pfaffenwaldring 55, \\ 7000 Stuttgart 80 , Germany
}

(Received 18 April 1991; accepted 4 June 1991)

\begin{abstract}
Studies on the spin distribution in the radical state of anthracene- $\sigma$ bridgeviologen supermolecules with different bridge units are reported. Electronnuclear double resonance experiments (ENDOR) were performed on electrochemically reduced molecules. Proton hyperfine coupling constants at different molecular sites were obtained and are discussed in detail. The experimentally determined values are compared with quantum chemical calculations of the INDO type. The observed spin distribution depends significantly on the type of the bridging unit. The relevance of the spin distribution for electron transfer in donor-bridge-acceptor molecular complexes is discussed.
\end{abstract}

\section{Introduction}

Intense research activity has emerged during the last decade on charge transfer (CT) processes in organic molecular complexes. Among others in particular biological and synthetical systems, like donor-bridge-acceptor (D-B-A) molecules attract considerable interest because of their advantages for a critical comparison of experimental data and theories of electron transfer (ET) processes [1-5]. Such D-B-A systems are suitable model compounds for solar energy conversion by a photochemical process where the donor unit acts as a photosensitizer and the acceptor unit as an electron carrier [5].

In recent years there is also a considerable effort to utilize molecular structures for application in molecular electronics [6]. One of the early proposals by Aviram and Ratner suggested that a unimolecular oriented film of organic molecules in the form of D- $\sigma-A$ (where D and A stand for electron donor and acceptor, respectively, with a saturated covalent $\sigma$ bridge) sandwiched between two metal films should act as an electrical rectifier [7]. Although this rectifier function has not been demonstrated to date it would if its works perform similar to semiconductor pn junction however reduced in size by a factor up to 200 compared to usual semiconductor structures [8]. There are several requirements on the $\sigma$ spacer unit. Among others, the $\sigma$ bridge must be long enough, in order to decouple the molecular energy levels of the donor and acceptor significantly. On the other hand the flexibility of the spacer unit should be rather small in order to prevent a U-shaped

$\dagger$ Present address: Institute for Polymers and Organic Solids, University of California, Santa Barbara, CA 93105, USA. 
molecular structure with strong through-space interaction between donor and acceptor [8].

It has been suggested recently to utilize such D-B-A 'supermolecules' as molecular information storage units [9]. The donor or equivalently the acceptor may be excited by light irradiation resulting in an excited state which permits electron transfer via the bridge molecule from the donor to the acceptor or hole transfer from the acceptor to the donor. The final state $\mathrm{D}^{+}-\mathrm{B}-\mathrm{A}^{-}$may be visualized as a logical ' 1 ' whereas the ground state $\mathrm{D}-\mathrm{B}-\mathrm{A}$ is assigned to the logical state ' 0 '. The higher energy of the $\mathrm{D}^{+}-\mathrm{B}-\mathrm{A}^{-}$state will force the system to decay very rapidly to the ground state due to tunnelling processes. The nature of the spacer between the donor and the acceptor units plays a fundamental role in the ET process. Besides the tunnelling matrix elements also the conformational relaxation of the molecular units in the $\mathrm{D}^{+}-\mathrm{B}-\mathrm{A}^{-}$state as well as the environmental reorganization in order to achieve an effective dielectric screening of the charged units are of paramount importance [9].

Moreover other effects like exothermicity of the reaction and solvent as well as the electronic states of the spacer unit of those D-B-A molecules play an important role in the electron transfer process. For the $\mathrm{Zn}$-Porphyrin-bicyclo-octyl-benzoquinone molecule as a photosynthetic model compound the increase of the aliphatic spacer length from 10 to $14 \AA$ (at fixed exothermicity, solvent and temperature) thus revealed a change of electron transfer rate from $10^{10} \mathrm{~s}^{-1}$ to $\leqslant 10^{7} \mathrm{~s}^{-1}$ [4]. Furthermore, considerably fast electron transfer (completed forward transfer within $0.5 \mathrm{~ns}$ ) is observed over long distances of saturated bridged molecules with high energy barrier height $(5 \mathrm{eV})$ and with a negligible probability of finding the electron in the spacer [10]. The electron transfer mechanism can be described as a long range electron tunnelling process [10]. Detailed theoretical condiderations show the critical dependence of the tunnelling matrix element on the distance between the donor and acceptor (e.g. on the effective length of the bridge unit) and on the symmetry of $\pi$ orbitals of the donor relative to the binding states of the bridge unit [11]. Recent optical studies demonstrate the effects of through-bond interaction of the donor and the acceptor in rigid $\sigma$ bridged $\mathbf{D}-\mathrm{B}-\mathrm{A}$ molecules with variation of the distance (length of the spacer) [5].

We have applied electron-nuclear-double-resonance (ENDOR) as a powerful method to monitor the spin distribution on a molecular system with unpaired electrons $[12,13]$. In solution, the hyperfine coupling tensor reduces to the isotropic hyperfine coupling (HFC) constant, leading for an electron spin $S=1 / 2$, coupled to $N$ nuclei with $I=1 / 2$ to $N$ pairs of ENDOR lines, each of them symmetrically spaced about the free nuclear Larmor frequency and separated by the HFC constant. From the HFC constants of the protons the spin density in the $\pi$ orbital at the neighbouring carbon site can be determined by simple relations [13].

In this work we report on studies of the spin distribution in $\sigma$-bridged $\mathrm{D}-\mathrm{B}-\mathrm{A}$ supermolecules with different bridge units consisting of anthracene and viologen as the donor and the acceptor, respectively. The hyperfine coupling constants of the electrochemically radicalized molecules, obtained from ENDOR experiments in solutions, depend on the bridge unit. Our results show a distortion of the spin distribution in the molecule depending on the nature and length of the bridge unit as well as on the symmetrical and/or asymmetrical linkage of the acceptor unit (D-B-A-B-D and D-B-A forms, respectively). The through-bond interaction between the donor and the acceptor units show up in the change of the spin density (e.g. HFC) on the bridge units. 
a)

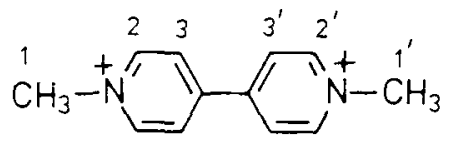

b)

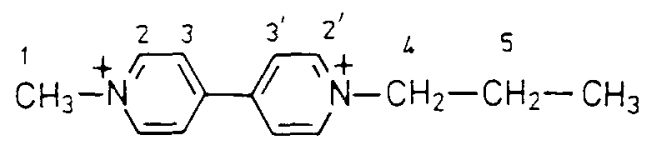

C)

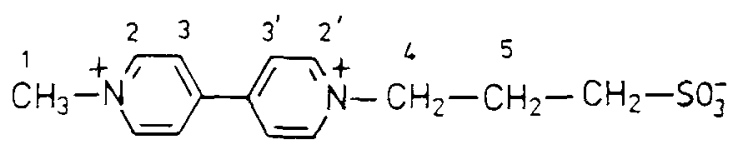

Figure 1. Viologen derivatives which were used as reference molecules. (a) 1, 1'-dimethyl-4, 4'-bipyridinium, (b) 1-methyl-1'-propyl-4, 4'-bipyridinium, (c) 1-methyl-1'-(3-sulfonatoproply)-4, 4'-bipyridinium (halfzwitterviologen). The numbers enumerate the protons with observed and/or calculated hyperfine coupling constants.

Also the INDO calculations display the same dependence on the bridge units as is observed experimentally. A comparison between the experimental results and the calculations will be made in the discussion section.

\section{Experimental}

For reference we have drawn some viologen derivatives in figures 1(a) to 1(c). A sketch of the structures of the molecules investigated here including the numbering of the different atomic sites is displayed in figure 2(a) to 2(d). Details of the synthesis and electrochemical studies will be published elsewhere [14]. Radical cations of the molecules are obtained by electrochemical reduction. The electrolyte used consists of tetrahydrofuran (THF) which was vacuum distilled over $\mathrm{CaH}_{2}$ and freeze cycled at least three times with about $0.1 \mathrm{M}$ dried tetrabutylammoniumperchlorate as the conduction salt. The home made electrochemical cell is attached to a vacuum line and filled with solvent by vacuum distillation. After electrochemical reduction of the molecules the radical solution is filled into the quartz ENDOR tube and sealed by melting. During the preparation process all contact to ambient conditions and especially to oxygen is thus avoided. A schematic view of the electrochemical cell is displayed in figure 3 . The electrochemical reduction is carried out potentiostatically at constant potential of $-700 \mathrm{mV}$ as opposed to a modified $\mathrm{Ag}-\mathrm{AgCl}$ reference electrode. Platinum electrodes are used as working and counter electrodes in the three electrode electrochemical setup with a Jaissle potentiostat.

The ENDOR equipment consists of a computer Bruker ER 200 D EPR spectrometer, a Bruker ER 411 VT temperature control unit, a Rohde and Schwarz SMG r.f. signal generator with an ENI A 500 amplifier and a home- made TM $_{110}$ ENDOR resonator. All ENDOR experiments were carried out at $220 \mathrm{~K}$ with magnetic fieldmicrowave frequency locked at resonance to eliminate possible drifts. After saturation of the ESR signal, an $\mathrm{rf}$ sweep is performed and changes of the ESR signal were detected.

\section{Experimental results}

ENDOR spectra of the molecules $2 \mathrm{a}$ and $2 \mathrm{c}$ are displayed in figure 4 . From the absence of the large couplings around 9 and $20 \mathrm{MHz}$ in the molecule $2 \mathrm{c}$ one can easily 
a)

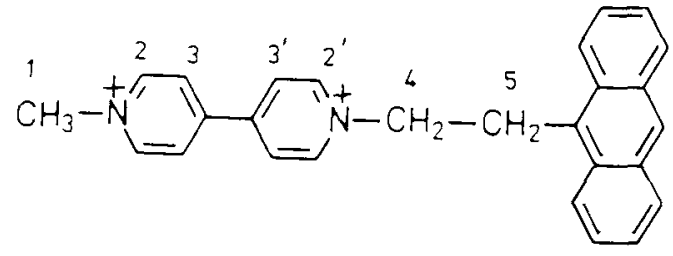

b)

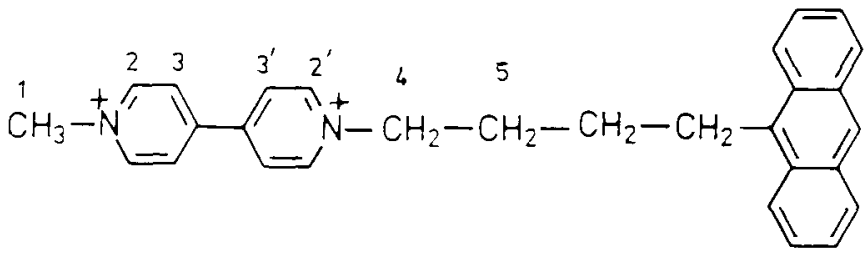

c)

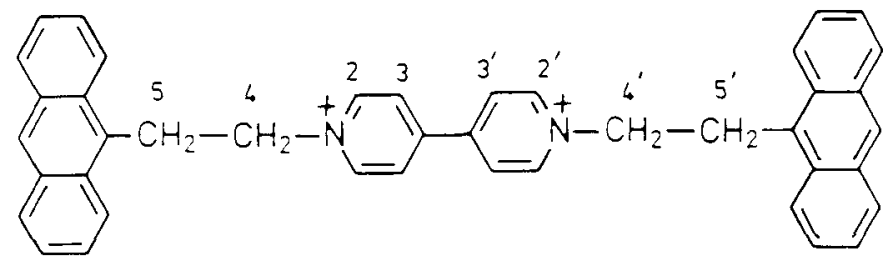

d)

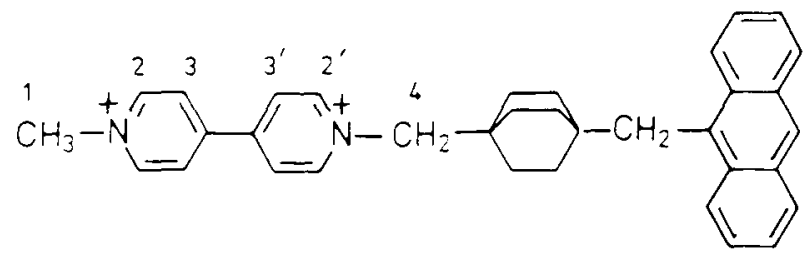

Figure 2. Molecules with donor- $\sigma$ bridge-acceptor based structures studied in this work. The numbers enumerate the protons with observed and/or calculated hyperfine coupling constants.

assign these couplings to the methyl group protons. The other ENDOR lines are identified and assigned to the alkyl substituted viologen [15-17]. Remarkable changes in the spectra are observed in the region $12-13 \mathrm{MHz}$ and $16-17 \mathrm{MHz}$ where the hyperfine couplings of the ring protons of the viologen unit are expected, thus, for molecule $2 \mathrm{a}$ three ENDOR lines are observable in that region, whereas for molecule $2 \mathrm{c}$ only two lines are resolved.

ENDOR spectra for the molecules $2 b$ and $2 d$ are displayed in figure 5. For molecule $2 \mathrm{c}$ the two lines of the ring proton couplings are much closer to each other than in molecule $2 \mathrm{~b}$ as can be seen from figure 4 . The couplings around 11 and $18 \mathrm{MHz}$ show differences between molecules $2 \mathrm{~b}$ and $2 \mathrm{~d}$. All HFC constants obtained from figures 4 and 5 are summarized in the table.

We have also observed hyperfine structure resolved ESR spectra of these molecules. The observed ESR spectra are consistent with the ENDOR spectra. We report here, however, only the ENDOR results, because of their superior resolution. We started a simulation of the ESR spectra using the HFC constants from the ENDOR experiments.

\section{Theoretical}

In order to compare the experimentally observed hyperfine interactions with the electronic states of the D-B-A complex we have performed INDO type quantum 


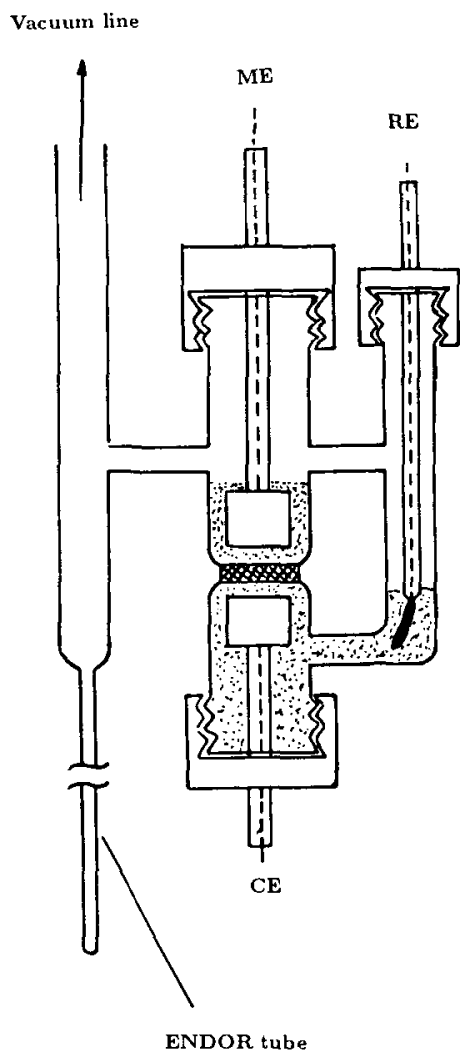

Figure 3. A schematic view of the ex situ electrochemical cell for radicalization of the molecules for ENDOR studies. WE, RE and CE represent working $(\mathbf{P t})$, reference $(\mathrm{Ag}-\mathrm{AgCl})$ and counter $(\mathrm{Pt})$ electrodes, respectively.

chemical calculations $[18,19]$. Our version of INDO was parameterized by Plato with the main emphasis for obtaining reliable proton hyperfine interactions in organic radicals. It has been proven to be successful even in the calculation of large molecules like the reaction centre of photosynthesis [13]. The INDO calculations were performed on a Hewlett Packard computer series 9000/840.

For the anthracene and the alkyl units we took standard geometries of totally aromatic and all trans structures, respectively. We assumed for the geometry of the viologen units coplanar aromatic structures with interannular ring separation of $1.46 \AA$ and $\mathrm{C}=\mathrm{C}, \mathrm{C}=\mathrm{N}$ bond lengths of $1.4 \AA$ with $120^{\circ}$. The $\mathrm{N}-\mathrm{CH}_{3}$ and $\mathrm{N}-\mathrm{CH}_{2}$ distances were chosen as 1.47 and $1.45 \AA$, respectively.

In table 1 the results of the INDO calculations for the proton hyperfine interactions are compared with the experimentally obtained data.

\section{Discussion}

We have summarized in table 1 all proton hyperfine coupling constants in $\mathrm{MHz}$ units. The theoretically calculated values are presented in parentheses. Let us first discuss the experimentally determined data.

The assignment of the HFC constants in a viologen radical state was performed by Clack et al. [16] earlier by using deuteration of selected ring proton sites. Thus, in 


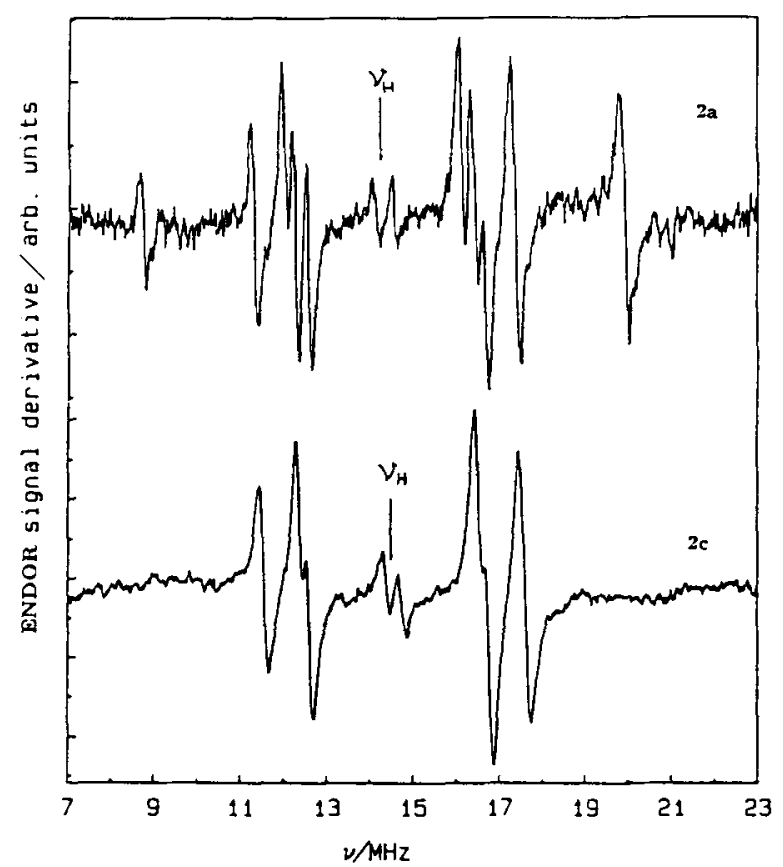

Figure 4. Proton ENDOR spectra of molecules $2 a$ and $2 c$ (figure 2).

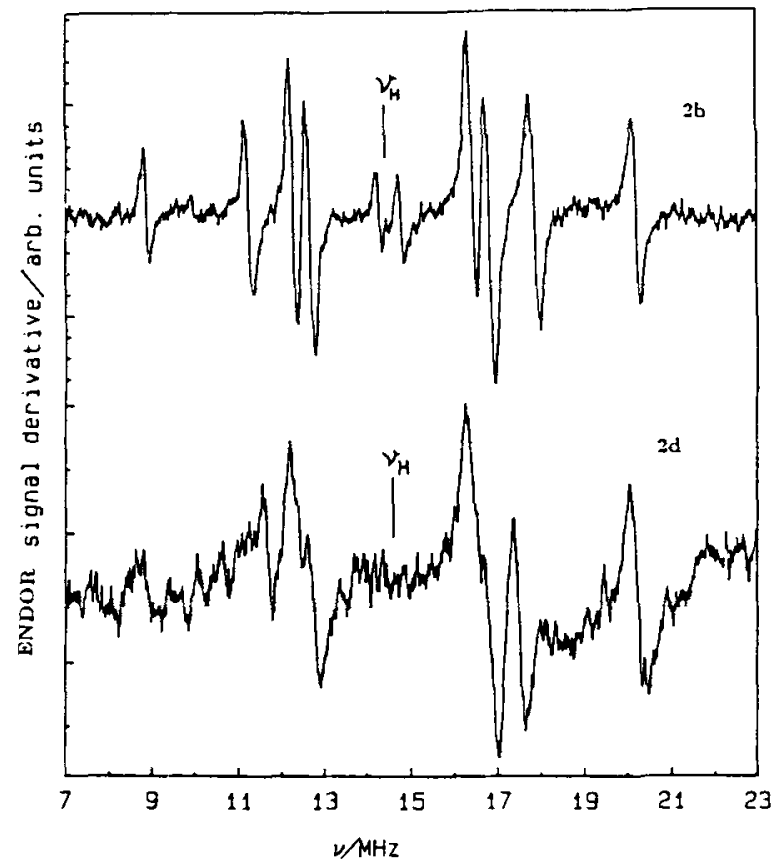

Figure 5. Proton ENDOR spectra of molecules $2 \mathrm{~b}$ and $2 \mathrm{~d}$ (figure 2). 
Table 1. Proton hyperfine coupling constants in $\mathrm{MHz}$ of the molecules as numbered in figures 1 and 2. Theoretical data from INDO calculations are represented by the values in parenthesis. In molecule $2 a$ symmetry breaking is assigned to positions 3 and $3^{\prime}$ by comparison with HFC of molecules $2 b$ and $2 c$. The sign of HFC's for the experimental part are chosen by comparison with the INDO calculations.

\begin{tabular}{|c|c|c|c|c|c|c|c|c|}
\hline & 1 & 2 & 3 & $3^{\prime}$ & 2 & 4 & 5 & Ref. \\
\hline 1a.) & $\begin{array}{l}11.46 \\
(8.13)\end{array}$ & $\begin{array}{l}-3.81 \\
(-4.75)\end{array}$ & $\begin{array}{l}-4.71 \\
(-1.60)\end{array}$ & $\begin{array}{l}-4.71 \\
(-1.60)\end{array}$ & $\begin{array}{l}-3.81 \\
(-4.75)\end{array}$ & & & $\begin{array}{l}\text { (16) } \\
\text { this work }\end{array}$ \\
\hline 1b.) & $\begin{array}{l}\text { I1.32 } \\
(8.33)\end{array}$ & $\begin{array}{l}-3.78 \\
(-5.13)\end{array}$ & $\begin{array}{l}-4.62 \\
(-1.43)\end{array}$ & $\begin{array}{l}-4.62 \\
(-1.98)\end{array}$ & $\begin{array}{l}-3.78 \\
(-3.96)\end{array}$ & $\begin{array}{l}6.33 \\
(4.43)\end{array}$ & $\begin{array}{l}-0.308 \\
(-0.56)\end{array}$ & $\begin{array}{l}{[21]} \\
\text { this work }\end{array}$ \\
\hline lc.) & $\begin{array}{l}11.19 \\
(9.16)\end{array}$ & $\begin{array}{l}-3.64 \\
(-i .27)\end{array}$ & $\begin{array}{l}-4.76 \\
(-0.40)\end{array}$ & $\begin{array}{l}-4.76 \\
(-2.91)\end{array}$ & $\begin{array}{l}-3.64 \\
(-1.85)\end{array}$ & $\begin{array}{l}7.56 \\
(3.99)\end{array}$ & $(-0.50)$ & $\begin{array}{l}17: \\
\text { this work }\end{array}$ \\
\hline 2a.) & $\begin{array}{l}11.17 \\
(8.36)\end{array}$ & $\begin{array}{l}-3.56 \\
(-5.34)\end{array}$ & $\begin{array}{l}-4.17 \\
(-1.32)\end{array}$ & $\begin{array}{l}-4.69 \\
(-2.06)\end{array}$ & $\begin{array}{l}-3.56 \\
(-3.77)\end{array}$ & $\begin{array}{l}6.11 \\
(5.32)\end{array}$ & $\begin{array}{l}-0.469 \\
(-0.63)\end{array}$ & $\begin{array}{l}\text { this work } \\
\text { this work }\end{array}$ \\
\hline 2b.) & $\begin{array}{l}11.33 \\
\langle 8.32\rangle\end{array}$ & $\begin{array}{l}-3.7 i \\
1-5.231\end{array}$ & $\begin{array}{l}-4.54 \\
(-1.37)\end{array}$ & $\begin{array}{l}-4.54 \\
(-1.99)\end{array}$ & $\begin{array}{l}-3.77 \\
(-3.92)\end{array}$ & $\begin{array}{l}6.61 \\
\{5.35\}\end{array}$ & $\begin{array}{l}-0.515 \\
(-0.59)\end{array}$ & $\begin{array}{l}\text { this work } \\
\text { this work }\end{array}$ \\
\hline 2c.) & & $\begin{array}{l}-3.93 \\
(-4.35 !\end{array}$ & $\begin{array}{l}-4.11 \\
(-1.79)\end{array}$ & $\begin{array}{l}-4.41 \\
(-1.79)\end{array}$ & $\begin{array}{l}-3.93 \\
(-4.35)\end{array}$ & $\begin{array}{l}6.05 \\
(5.48)\end{array}$ & $\begin{array}{l}-0.369 \\
(-0.65)\end{array}$ & $\begin{array}{l}\text { this work } \\
\text { this work }\end{array}$ \\
\hline 2d.) & $\begin{array}{l}11.54 \\
(8.41)\end{array}$ & $\begin{array}{l}-3.54 \\
(-5.37)\end{array}$ & $\begin{array}{l}-4.54 \\
(-1.32)\end{array}$ & $\begin{array}{l}-4.54 \\
(-2.09)\end{array}$ & $\begin{array}{l}-3.54 \\
(-3.72)\end{array}$ & $\begin{array}{l}5.89 \\
(5.31)\end{array}$ & & $\begin{array}{l}\text { this work } \\
\text { this work }\end{array}$ \\
\hline
\end{tabular}

methyl substituted viologen systems, the magnitude of the HFC constants of the ring protons in position 3 is found to be greater than the ring protons in position 2. Interestingly enough a phenyl substitution does reverse this relation. This phenomenon is assumed to arise from geometrical repulsion effects of the ring protons [16]. Additionally, the nature of the substituent on the viologen molecule also influences the spin distribution drastically [21]. An electron donor unit, directly attached to the viologen molecule, thus causes symmetry breaking among the ring proton couplings, whereas no such effect is observed by asymmetrical alkyl substitution [21].

The effect of anthracene attachment to the alkyl viologen is clearly observed in our data. Note that the molecule 1c (halfzwitterviologen) with an asymmetric alkyl substitution shows a symmetric four-fold degeneracy [17] of the ring proton hyperfine constants, whereas the removal of this symmetry is observed here when anthracene is attached to the alkyl chain, i.e. the $\pi$ electron cloud of anthracene couples to the spin distribution on the viologen. This is observed clearly for the shortest $\sigma$ bridge $\left(2 \mathrm{CH}_{2}\right)$ at molecule $2 \mathrm{a}$ where three ring proton couplings instead of two are observed (figure 4).

The longer alkyl bridge $\left(4 \mathrm{CH}_{2}\right)$ in molecule $2 \mathrm{~b}$ represents better isolation of the radical spin on the viologen from the anthracene. There is, however, the possibility for folding over due to the larger flexibility of this bridge. The all syn formation of the alkyl bridge, however, is not possible in molecule $2 \mathrm{~b}$ because of the steric hindrance of the anthracene and viologen units. Thus, the HFC constants observed are very close to the methyl viologen radical [16] but still a considerable HFC constant is observed at the second $\mathrm{CH}_{2}$ unit.

The bicyclo-octane spacer in the molecule $2 \mathrm{~d}$ is a good spacer with a strong decoupling of the donor and acceptor. The HFC constants observed here are also close to the methyl viologen radical. The remarkable difference between molecule $2 b$ 
and $2 \mathrm{~d}$ is the $\mathrm{HFC}$ of the first $\mathrm{CH}_{2}$ unit, where a larger coupling is observed in the butyl bridged molecule compared to the bicylo-octane bridged one. The latter bridge type is, however, to be preferred as far as donor acceptor isolation is concerned because of its restricted flexibility.

The influence of the anthracene unit on the spin distribution at the viologen is evident from the comparison of the HFC constants between molecule $2 \mathrm{a}$ and $2 \mathrm{c}$ (figure 4). Due to the symmetrical substitution, the splitting of the ring proton couplings into three ENDOR lines is removed and four-fold degeneracy is reestablished again. Additionally the difference in magnitudes of the two different ring proton HFC couplings is smoothed by the symmetrical perturbation of the anthracene units.

The intramolecular charge transfer reactions in the D-B-A system depend significantly on the nature of the bridge unit as stated above. From our results, the strong coupling of the donor $\pi$ system through a $2 \mathbf{C H}_{2}$ bridge to the acceptor spin distribution indicates the appreciable matrix element for spin transfer. Both the electron transfer and the spin transfer depend on the corresponding matrix elements between different molecular orbitals. A short lifetime of a $\mathrm{D}^{+}-\mathrm{B}-\mathrm{A}^{-}$excited state in a supermolecule with such a spacer has therefore to be expected. Even in the butyl bridge the large coupling of the second $\mathrm{CH}_{2}$ unit is an indication of appreciable backtransfer. The butyl bridge seems to be more isolating, although there is a strong possibility for backfolding, leading to through space interaction. The strongest isolating bridge unit is obviously the bicyclo-octane in the molecule $2 \mathrm{~d}$, although a slight loss of spin density on the first $\mathrm{CH}_{2}$ unit (see table 1) is observed which might hint at a U-shaped structure causing a through space tunnelling. Strong fluorescence quenching was observed in these molecules which is also an indication for rapid electron transfer [24].

From table 1 it is evident that the calculated hyperfine coupling constants obtained from INDO type calculations (data are in parentheses) are consistently smaller than the experimental data. Nevertheless the calculated data do reflect the trend previously discussed for the experimental data. There is, however, a fundamental discrepancy between the theoretical and experimental data which was observed already before, namely the interchange of the values for the ring protons at the viologen molecule [16]. Detailed INDO studies and comparison with results on different substituted viologens show this discrepancy arising from structural distortions in the radical state as discussed in a separate publication [22]. Otherwise the INDO calculations do reproduce the trend that the largest hyperfine interactions is due to the methyl group caused by hyperconjugation. The first $\mathrm{CH}_{2}$ group of the alkyl chain still has a large HFC constant, whereas the next $\mathrm{CH}_{2}$ group in the alkyl chain has a HFC which is reduced by about an order of magnitude with respect to the first one, consistent with the experimental observation. This also reflects the order of magnitude reduction in the tunnelling matrix element for electron transfer in each $\mathrm{CH}_{2}$ group in an alkyl chain [23].

In general the INDO calculations show a higher sensitivity to symmetry breaking. Whereas the experimental data for molecule $2 b$ show no symmetry breaking, a fact which we attribute to the strong decoupling of the anthracene from the viologen by the butyl group, the INDO calculation does show a symmetry breaking for molecule $2 \mathrm{~b}$ and similarly for molecule $1 \mathrm{~b}$ and $\mathrm{Ic}$. It is obvious, however, that the attachment of the anthracene molecule result in an enhanced symmetry breaking also for the INDO calculations as is evidenced by comparing the HFC constants of molecules $1 \mathrm{~b}$ and $2 \mathrm{a}$. Finally, the poor quantitative agreement between the calculated and experimentally observed HFC constants in viologen systems is found to be due to huge 
influence of slight geometrical changes in the INDO calculations on the hyperfine coupling constants.

We are grateful to M. Plato (FU Berlin) for making his version of the INDO program available. This work is supported by the Deutsche Forschungsgemeinschaft SFB 329.

\section{References}

[1] Joran, A. D., Leland, B. A., Geller, G. G., Hopfield, J. J., and Dervan, P. B., 1984, J. Am. Chem. Soc., 106, 6090.

[2] Miller, J. R., Calcaterra, L. T., and Closs, G. L., 1984, J. Am. Chem. Soc., 106, 3047.

[3] Heitele, H., and Michel-Beyerle, M. E., 1985, J. Am. Chem. Soc., 107, 8286.

[4] Joran, A. D., Leland, B. A., Felker, P. M., Zewail, A. H., Hopfield, J. J., and Dervan, P. B., 1987, Nature, 327, 508 .

[5] Noda, S., Hosono, H., Okura, I., Yamamoto, Y., and Inoue, Y., 1990, J. Chem. Soc. Faraday Trans., 86, 811.

[6] CaRTeR, F. L., (ED.), 1987, Molecular Electronic Devices I/II, (Marcel Dekker).

[7] Aviram, A., and Ratner, M. A., 1974, Chem. Phys. Lett., 29, 277.

[8] Metzger, R. M., and Panetta, C. A., 1987, Molecular Electronic Devices II, edited by F. L. Carter (Marcel Dekker).

[9] Mehring, M., 1989, Springer Series in Sol. State Sci, 91, 242.

[10] Calcaterra, L. T., Closs, G. L., and Miller, J. R., 1983, J. Am. Chem. Soc., 105, 670.

[11] Beratan, D. N., 1986, J. Am. Chem. Soc., 108, 4321.

[12] Grupp, A., and Mehring, M., 1990, Advances in Pulsed and Continuous Wave Electron Spin Resonance, edited by L. Kevan and M. K. Bowman (John Wiley), Chap. 4.

[13] Kurreck, H., Kirste, B., and Lubitz, W., 1988, Electron Nuclear Double Resonance Spectroscopy of Radicals in Solutions, $(\mathrm{VCH})$, p. 90.

[14] EfFenberger, F., Götz, G., and BäUerle, P., in preparation.

[15] Johnson, C. S., and Gutowsky, H. S., 1963, J. Chem. Phys., 39, 58.

[16] Clack, D. W., Evans, J. C., Obaid, A. Y., and Rowlands, C. C., 1983, Tetrahedron, 39, 3615.

[17] ZwEYgart, W., 1989, Thesis, University of Stuttgart.

[18] Pople, J. A., and Beveridge, D. L., 1970, Approximate Molecular Orbital Theory, (McGraw Hill).

[19] Pople, J. A., Beveridge, D. L., and Dobosh, P. A., 1967, J. Chem. Phys., 47, 2026.

[20] Götz, G., 1990, Thesis, University of Stuttgart.

[21] Evans, J. C., Morris, C. R., and Rowlands, C. C., 1987, Tetrahedron, 43, 5329.

[22] Sariciftci, N. S., Werner, A., and Mehring, M., 1992, Molec. Phys., 75, 1269.

[23] Beratan, D. N., Onuchic, J. N., and Hopfield, J. J., 1987, J. Chem. Phys., 86, 4488.

[24] Weigand, B., private communication. 